\title{
Italian National Vegetation Database (BVN/ISPRA)
}

\author{
Laura Casella, Pietro Massimiliano Bianco, Pierangela Angelini \& Emi Morroni
}

\begin{abstract}
The BVN project aims to develop a Georeferenced National Vegetation Database, by digitizing and compiling vegetation plot data, from published and unpublished sources. The aim is to provide data for vegetation mapping projects and/or nature conservation and research programs (NATURA 2000, land planning, biodiversity studies, etc.). This geodatabase is an initiative of the National Institute for Environmental Protection and Research (ISPRA), a public body reporting to the Ministry of Environment, performing technical, scientific and research activities on environmental issues. A key tool in the activity of the Institute is the "Nature Map" Project, the vegetation map of Italy at the scale 1:50.000 using the Corine Biotopes/Palaearctic/EUNIS classifications. In order to complete the project, since 2004 a large number of vegetation-floristic data have been collected on the national territory (currently the project has covered half of the territory) on ground control points and from literature sources and managed in a Geographic Information System. This geodatabase constitutes the core of the project; the final format will be available online for consultation and upon request for scientific purposes. The BVN is developed using TURBOVEG, in agreement with the activities of the European Vegetation Survey. The geodatabase currently comprises 4400 relevés and it is growing rapidly. This report describes the available content in the Italian National Vegetation Database (BVN/ISPRA; GIVD ID EU-IT-010).
\end{abstract}

Keywords: EUNIS/Palaearctic classification; Geodatabase; Italy; vegetation mapping

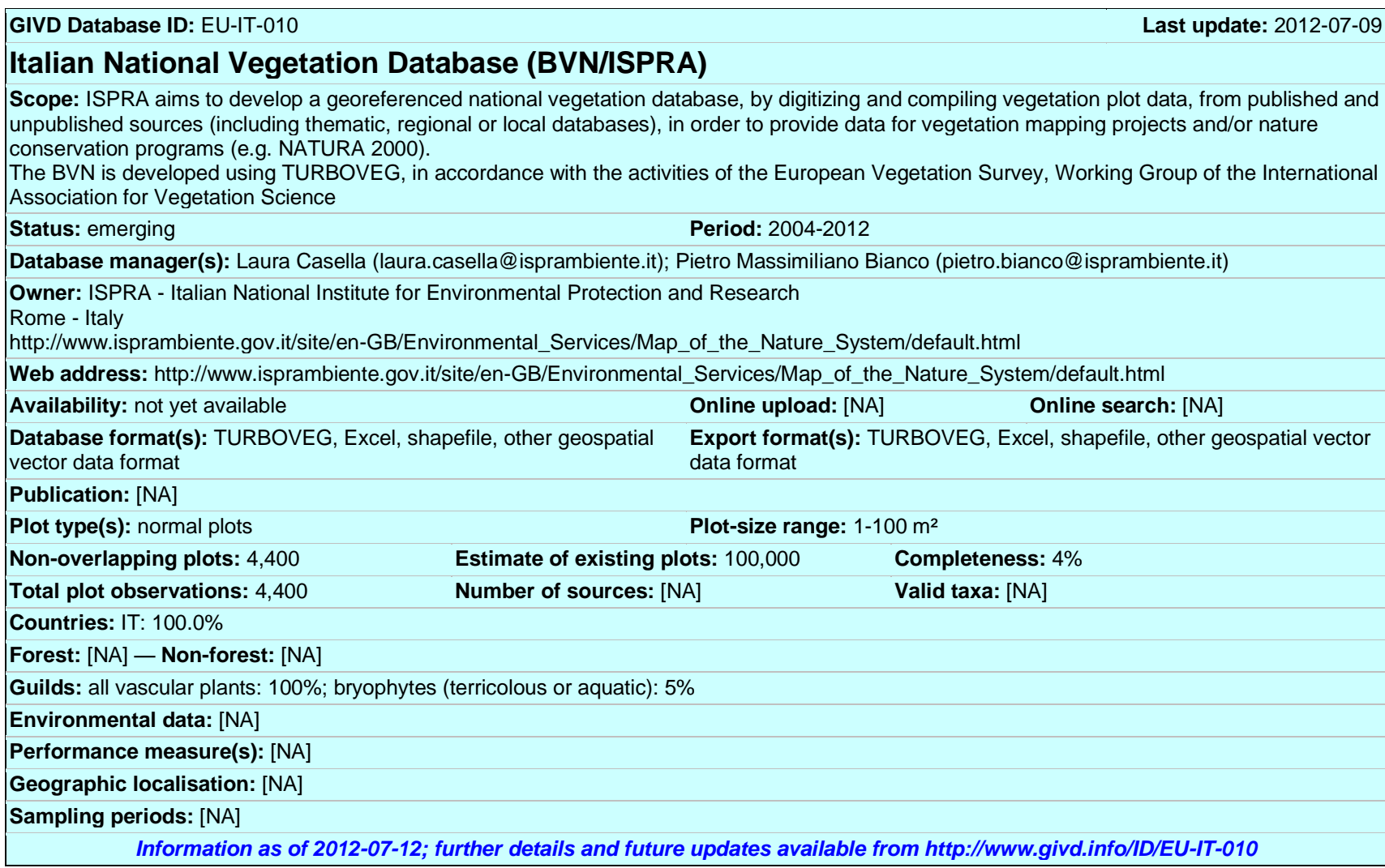

Laura Casella* (laura.casella@isprambiente.it), Pietro Massimiliano Bianco (pietro.bianco@isprambiente.it), Pierangela Angelini (pierangela.angelini@isprambiente.it), Emi Morroni (emi.morroni@isprambiente.it)

Nature Protection, ISPRA - Italian National Institute for Environmental Protection and Research, Via Vitaliano Brancati, 60, 00144 Rome, ITALY

*Corresponding author 